\title{
On the chaotic nature of Electro-Discharge Machining
}

\begin{abstract}
The long-accepted thermoelectric model for electro-discharge machining is being brought into question. Several experimental facts prompt the proposal of a new theory, based on the effect of gap pollution on the ignition of discharges. The first experimental proof comes from the recently reported observation of debris chains and clusters. In this view, each step of the process depends on the previous ones through a deterministic relation, even if the overall evolution is unpredictable. The paper establishes mathematical grounds for the abovementioned intuitions, by setting up and solving a recursive equation for the machining energy employed at each discharge event. By means of numerical and algebraic tools the above equation is studied and shows a chaotic evolution similar to that of the Logistic map. Results reconcile the apparent paradox between deterministic nature and stochastic localization of the discharges and introduce a description of the chaotic dynamics of electro-discharge processes.
\end{abstract}

Keywords: electro-discharge machining, debris bridges, chains/clusters, chaos theory, numerical and algebraic computing, problem solving scientific environment

\section{Introduction}

The traditionally accepted thermoelectric theory for Electro-Discharge Machining (EDM) is described in the review by Ho and Newman [1] and taken as the ground for many models, as exemplified in the critical work by Yeo et al. [2]. The basic assumption in all of these studies is that localized sparks (electrical discharges) are initiated by ion action across the inter-electrode gap, when the strength of the dielectric fluid is overcome. A plasma channel is thus generated, which melts material at the surface of each pole, both workpiece and tool. When the pulsating current supply is turned off, the temperature suddenly drops, the plasma channel implodes and the molten material is flushed in the form of microscopic debris.

Despite the fact that EDM is a diffused technology, many authors agree with Yeo and colleagues [3] on the lack of a simple and comprehensive theory explaining the material removal process in detail. What is more, a scientific debate has recently arisen that questions the above theory on the basis of experimental observations collected and reviewed by Schumacher [4,5]. Ignition delay, gap widening, minimum current and influence of off-time are only the major hints that have led to speculate a new model for discharge ignition, by evaporation of particle bridges. Further details on experimental facts against the thermoelectric theory are collected in the Appendix section of this paper. Schumacher infers that debris aligns under the influence of the electric field and the obstruction by the gas bubbles; the bridges 
short-circuit the gap and vaporize by Joule heat, originating the plasma channel. This new model for the ignition of discharges complies with more recent observations of gas bubbles performed by Schulze et al. [6] and is gaining acceptance in the literature. Luo recognizes a dominant function of debris in discharge transitivity [7].

The authors of the present paper embrace Schumacher's theory: the first evidence of the presence of debris bridges in electro-discharge (ED) drilling of a ceramic composite has been recently provided [8], while here consequences of Schumacher's theory are rendered in terms of a mathematical model. A speculative consequence of the new discharge model is that the process may evolve toward a chaotic behavior when the debris contamination increases. The complexity of a process with many variables and superimposing effects has been traditionally combined with a stochastic nature, for example by Yeo and colleagues and by Pandit and Rajurkar [3,9]. In many studies deterministic models coexist with a stochastic occurrence of discharges, which brings to an apparent paradox. Kunieda and colleagues started to propose a chaotic rather than stochastic location of discharges [10,11]. This paper aims to overcome the dichotomy between deterministic and stochastic phenomena, by means of a mathematical model described by a deterministic recursive equation whose evolution depends on an unpredictable current state, leading to a computer assisted proof of the possibility of a chaotic evolution.

\section{Working energy, spark quality and ignition phenomena in EDM}

Energy partition and quality of electrical discharges are briefly surveyed in this section, in order to lay the foundation for the proposed model.

The literature is not unanimous on how energy distributes in the discharge zone. A shared starting point is obviously the relevance of the thermophysical properties of workpiece and tool materials, i.e. thermal diffusivity and conductivity, specific heat, melting and boiling temperatures. Most authors model the energy transfer by conduction to tool and workpiece and neglect heat losses by convection or radiation [3,121], which is instead the focus of Adineh's work [13]. Jilani and Pandey confirm the irrelevance of dissipative terms, calculating that as much as $90 \%$ of the total energy, liberated during a discharge, is conducted to the electrodes [14]. Part of this energy goes into melting and evaporation of material, but most of the material itself re-solidifies. Van Dijck estimates the ratio of the ejected-to-melted volume to be as little as $1 \%$ to $10 \%$ [15]. Patel et al. move the focus from energy to power and affirm that a small constant fraction of the total power is transferred to the anode, irrespectively of pulse current and duration [16]. A scheme of the spark energy distribution among anode, cathode, debris, dielectric fluid and radiated surrounding is shown in Figure 1, based also on the work by Xia et al. [17]. 


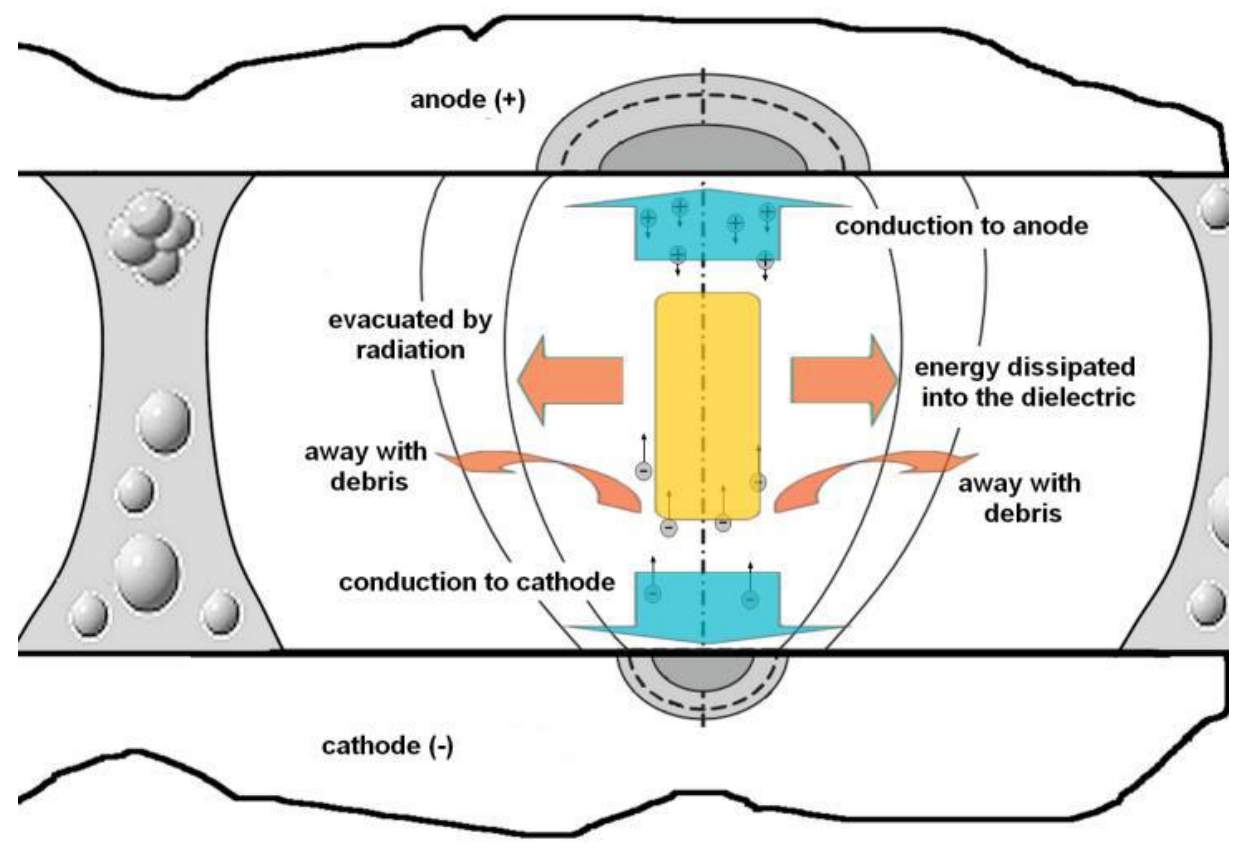

Figure 1. Energy distribution in the discharge area.

The sum of the terms in Figure 1, that is the total energy of a discharge, is a function of discharge voltage $V(t)$, current intensity $I(t)$ and pulse duration (in particular, we will denote $t_{o n}$ the pulse-on duration; see Formula (1) and Figure 5 in the following Section 3). However, discharges are complex events, whose evolution under many influences is not easily predictable. Even for constant process settings and a stable machining process, different types of discharges occur. The actual trend of voltage and current during a discharge, as well as the pulse duration and discharge energy, can markedly vary under different discharge conditions. Kunieda and colleagues offer an accurate view of the various scenarios [11]. Several authors dealt with the classification of discharges in EDM, mainly intended for process control. As an example, Behrens et al. and Ginzel et al. study the impulse parameters in the case of normal/abnormal sparks, including the case of arcing, and their influence on discharge energy $[18,19]$. Other authors distinguish the voltage and current curves of four pulse types: short circuits, arcs, normal discharges and open circuits [20-22]. To our purpose, a similar categorization is assumed and illustrated in Figure 2. 


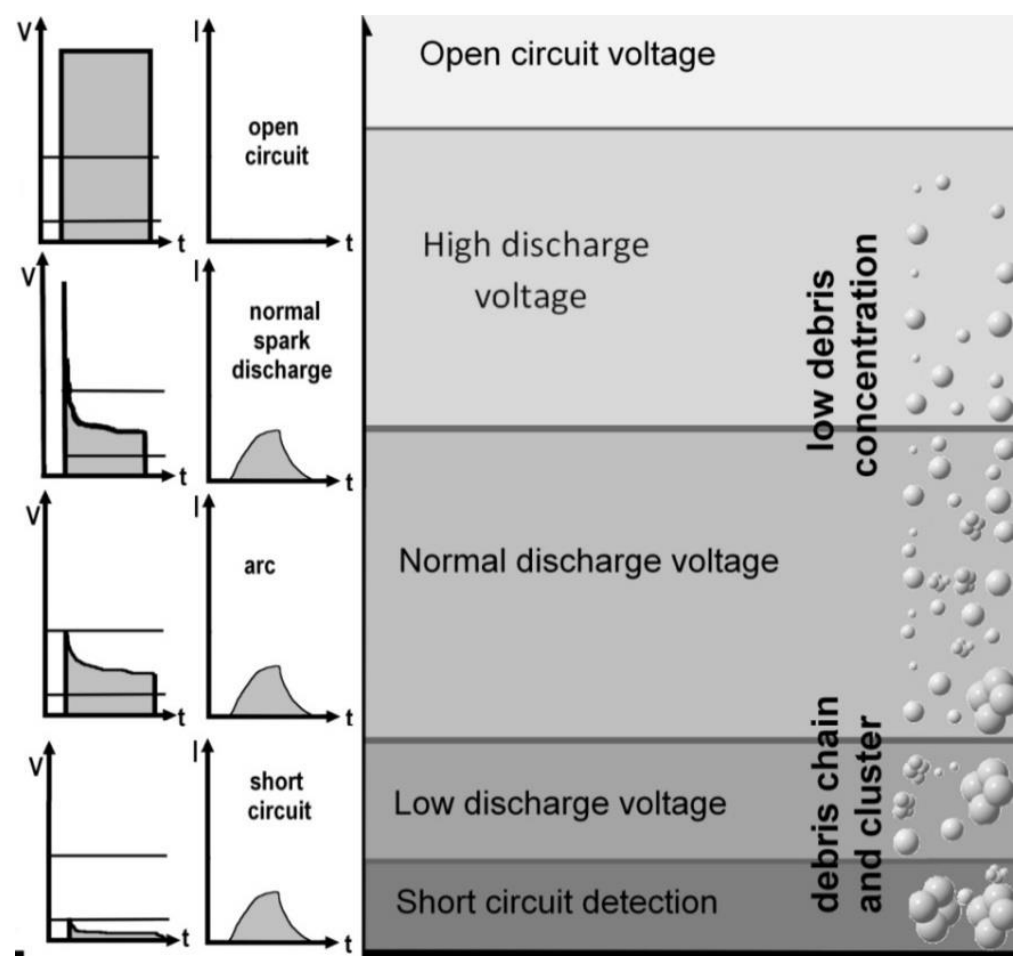

Figure 2. Categorization of pulse types: short circuits, arcs, normal discharges and open circuits

Arcs are low-voltage discharges with low available working energy. The establishment of arcing conditions cannot be easily recognized [11]. It is known, however, that the quality of a discharge, and hence its efficiency, is to a great extent determined by the characteristics of the dielectric liquid. Frei et al. considered that dielectrics are subject to varying contamination conditions during the spark erosion process [23]. Chemical decomposition and cracking of the dielectric can eventually add their effect to that of debris. Kunieda et al. offer an illuminating review of the complexity of gap phenomena [11]. The concentration of suspended particles and bubbles is an extremely sensitive parameter for the initiation of a discharge. Discharges occur after an activation time, called ignition delay time, which reflects the gap conditions: for example, it decreases for decreasing gap width and increasing debris contamination. A short ignition delay results in a lower average gap voltage. As a consequence, it seems logical that an arc is a product of the removal history taking into account the previous discharges. Schumacher is the first to disagree with the traditional theory of discharge ignition by ion action and speculates that the discharge event starts with particle bridges evaporating through excessive current, or by ionization across the short residual distances between the particles [5]. Gas bubbles, proven to persist over the pulse sequence [6], hamper debris flushing. The actual gap width depends on the average gap contamination with gas bubbles and remaining debris from earlier sparks, which define the new discharge area. Discharge events, thus, typically move in clouds. Material removal occurs by evaporation during the ignition phase and, later, by ejection of molten material due to instantaneous boiling at the discharge spots. 


\section{Experimental evidence of discharge ignition via debris bridges}

In our study, the new theory of ignition via particle bridges is addressed through experiments of small-hole ED drilling on a $72 \mathrm{wt} \% \mathrm{Al}_{2} \mathrm{O}_{3}-28 \mathrm{wt} \% \mathrm{TiC}$ composite as in [24]. Tests are carried out using pure copper tubular electrodes and fresh water-soluble dielectric fluid. Drilling operations are performed as in [8]. Machining debris is collected and investigated in order to study the mechanisms of material removal. Specifically, debris is observed by SEM, as soon as they are collected and, as well, after sectioning. Inspection of the debris morphology is then refined by means of dual beam SEM/FIB (focused ion beam) equipment (FEI StrataTMDB235), which allows for in-chamber sectioning and immediate observation. Particle dimensions, granulometric distribution and hollowness are measured by image analysis. The values of apparent density are confirmed by mercury porosimetry. The minimum gap dimension is estimated by subtracting both the thickness of deposits on the electrode and the height of peaks on the eroded surface from the measurements of overcut. Through the quantification of debris dimension and hollowness, the number of particles produced, within a certain volume in the gap, is calculated and contrasted with the conditions of densest sphere packing. Even with an over-conservative estimate, results prove that the number of particles in the gap is statistically far sufficient for debris bridges to form. Experimental confirmation is found by gathering ample evidence of the presence of debris bridges. This is the first experimental proof of chains and clusters of debris, new to literature. Debris hollowness and necking are evident in Figure 3. Necks are also clearly visible in the SEM images of debris chains and clusters in Figure 4. The observation of debris chains, with distinct necks, is an irrefutable proof of the role of debris in the ignition of discharges. As a consequence, a new model for electro-discharge processes can be postulated, where discharge ignition at each step depends on the contamination in the gap derived from the previous steps. The process develops from each step to the next one following deterministic rules, but since the gap state at each moment is not known, the overall outcome is unpredictable. On this basis, a recursive deterministic model is proposed in Section 4.

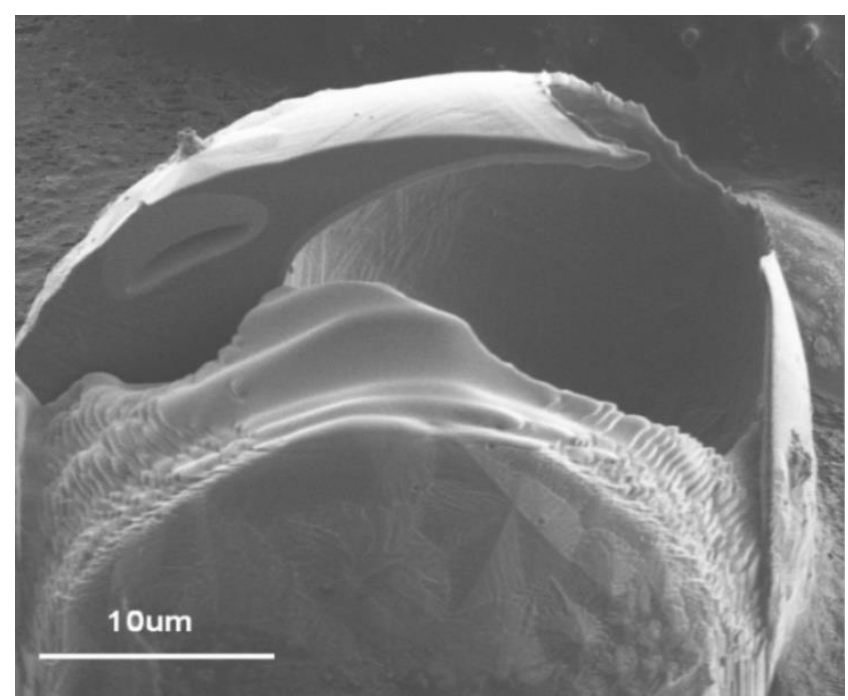

Figure 3. FIB-sectioned debris produced in small-hole ED drilling on a $72 \mathrm{wt} \% \mathrm{Al}_{2} \mathrm{O}_{3}-28 \mathrm{wt} \%$ TiC composite. 


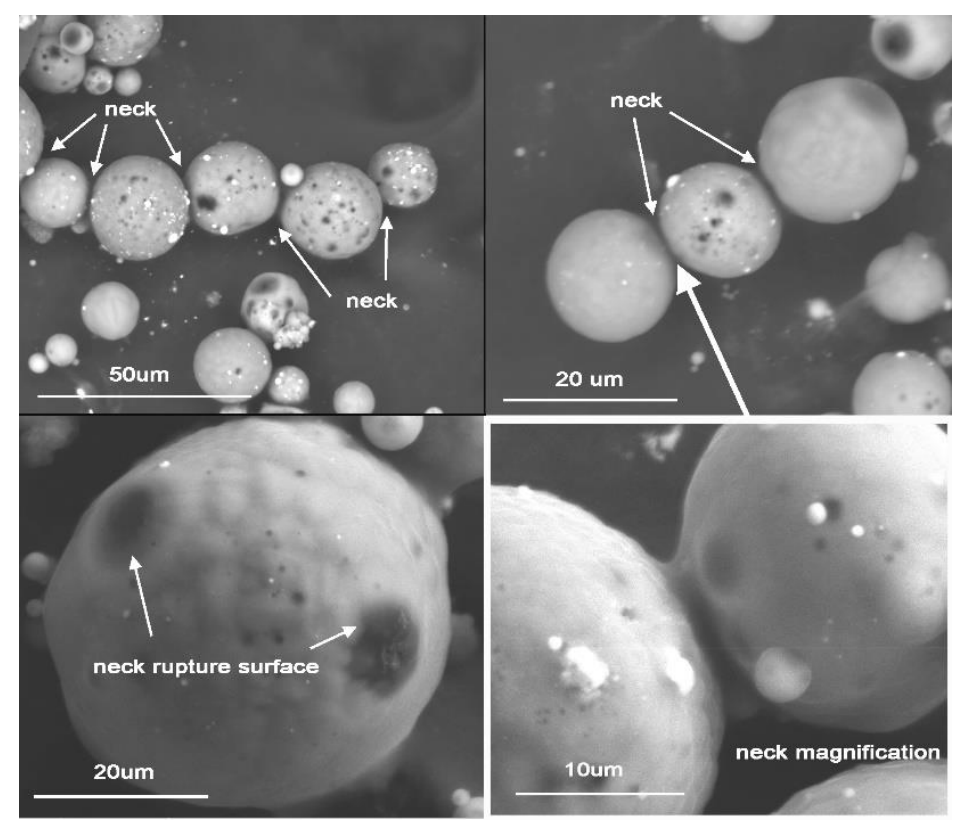

Figure 4. Examples of debris chains and clusters produced in small-hole ED drilling on a $72 \mathrm{wt} \% \mathrm{Al}_{2} \mathrm{O}_{3}-28 \mathrm{wt} \% \mathrm{TiC}$ composite.

The main points surveyed so far can then be summarized as follows.

- A share of the total discharge energy is available for material removal.

- Total working energy depends on discharge type.

- Discharge quality is influenced by gap state.

- Gap contamination is determined by previous spark history.

- Material removal mechanism is initial-condition dependent.

- Electro discharge machining has a non-linear nature [17].

- $\mathrm{EDM}$ is a recursive process.

These considerations suggest the need for a new model for electro-discharge processes founded on recursive laws and chaos theory.

\section{EDM as a recursive and deterministic model}

The total discharge energy or available working energy $E_{a w}$ in EDM is defined as in $[25,26]$ :

$E_{a w}=\int_{0}^{t_{o n}} V(t) I(t) d t$

Consider $E_{a w}$ to be calculated in the ideal condition of uncontaminated gap, where discharges are initiated by ion action, then $E_{a w}$ represents the maximum possible discharge energy. In all the actual contaminated conditions, the discharge energy will be lower than the ideal upper limit $E_{a w}$. In addition, the total available energy distributes in the gap area into 
several terms as sketched in Figure 1; therefore only a share of the total energy, namely the machining energy $\left(E_{m}\right)$, is available at the electrodes and goes into material removal.

Let us define the ratio $(E)$ between the machining energy and the maximum available working energy: $E$ indicates the percentage of the maximum energy that is effective for melting material. $E$ is a non-dimensional index of the efficiency of a discharge:

$E=\frac{E_{m}}{E_{a w}}, \quad 0<E<1$

The value of $E$ at each step depends on the energy at the previous discharge. If $E_{m}$ had a high value at the $\mathrm{k}^{\text {th }}$ step (that is to say that $E_{k}$, in Formula (3), also had a high value), then much debris will pollute the dielectric and there will be a high probability for chain and cluster formation. The $(\mathrm{k}+1)^{\text {th }}$ discharge will thus fall into one of the two cases shown in the lowest part of Figure 2, i.e. it will either be a low-voltage discharge or it will be moving towards short-circuiting conditions, with very little material removal. As a consequence, $E_{k+1}$ will be low. On the contrary, if the efficiency of the previous discharge was low, the gap will be little contaminated and a high discharge efficiency will be obtained at the current step. Gas bubbles in the dielectric act as local obstacles to debris flushing, so they increase gap pollution in selected areas and hamper the ignition of high-energy discharges at the next step. The actual gap width depends on the average gap contamination with gas bubbles and remaining debris from earlier sparks, which define the new discharge area.

This effect may be modeled by a recursive equation:

$E_{k+1}=\gamma\left(1-E_{k}\right)$

The effect of gas bubbles can be described with a decrease of $\gamma$. Moreover, the coefficient $\gamma$ must take into account an additional effect on the total energy, produced during a discharge, that is due to the actual time available for material removal during each pulse. The parameter $\gamma$ represents a term whose trend is opposite to that of the factor (1- $\left.E_{k}\right)$, i.e. $\gamma$ increases for increasing discharge efficiency at the previous k-th step, as explained in the remaining of this section. Each EDM cycle consists of a pulse-on time $\left(t_{o n}\right)$ and a pause time, i.e. a time interval $\left(t_{o f f}\right)$ between two successive sparks, as schematized in Figure 5. 


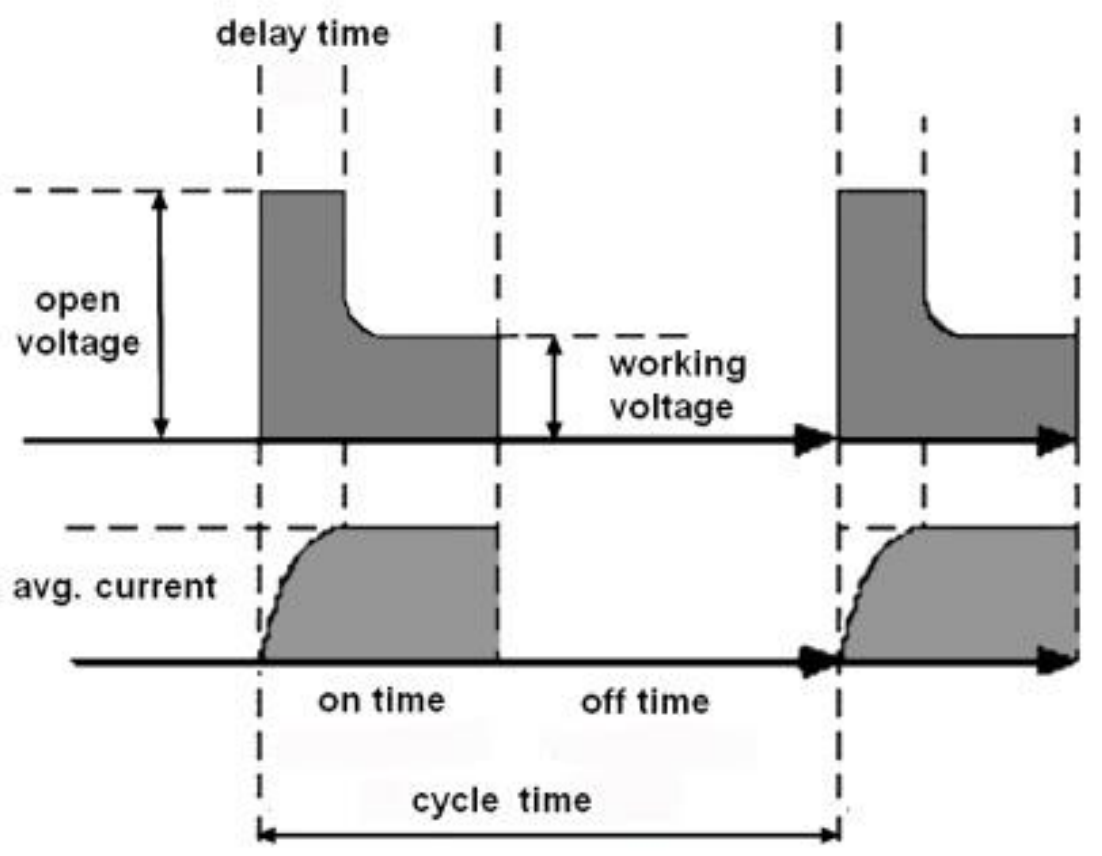

Figure 5. Scheme of EDM pulse times.

The duty ratio $(\tau)$ is usually defined as the percentage of pulse duration relative to total cycle time $\left(t_{o n}+t_{\text {off }}\right)$. Yet, the actual time available for material removal differs from $\mathrm{t}_{\mathrm{on}}$, due to the ignition delay time $t_{\text {delay. }}$. The actual duty ratio can be defined as:

$\tau_{\text {eff }}=\frac{t_{o n}-t_{\text {delay }}}{t_{o n}+t_{o f f}}$

From the discussion in Section 2, if ignition via particle bridges is accepted, recall that a strong gap contamination fosters discharge readiness and causes a shorter delay time. As a consequence, the actual duty ratio increases in accordance with the efficiency $E_{k}$ of the previous discharge, due to the abundance of debris. If $\tau_{\text {eff }}=0$, there is no discharge and the gap remains in the same state as at the previous step. If $\tau_{e f f}=1$, there is a short circuit and little material is removed.

Summing up these considerations, it is obtained that, in Formula (3), $\gamma$ should increase for increasing $E_{k}$ and reflect the influence of debris on the ignition delay time.

In a previous paper, the role of debris in discharge ignition and the conditions for bridge formation was analyzed [8].

Debris dimension, relative to gap width, determines the occurrence of bridges and hence the time required for discharge ignition. Based on these experimental findings, here the authors propose that $\gamma$ is proportional to the debris radius. In an attempt to build an energetic model, conjectures are formulated to relate the particle dimension to the energy index $E_{k}$. Let us consider the formation of debris particles, when part of the material that is melted during a discharge solidifies into debris. A reasonable assumption is that debris solidification occurs by heterogeneous nucleation. The motivation 
for this hypothesis lies in the presence of solid surfaces, solid phases and impurities that can potentially act as nucleants, thus reducing the free-energy barrier for nucleation. If Gibbs free energy is considered, particle formation occurs by growth of stable nuclei driven by two competitive factors, i.e. the volume free energy and the interfacial energy. If a nucleus can attain a size that exceeds the critical radius, then it grows while the total free energy decreases, since the (favourable) volume term overtakes the (unfavourable) surface term. Hence, during debris formation, the free energy decreases in accordance with the third power of the particle radius. In a reverse perspective, this suggests that debris radius may be related to the cube root of energy variation. The authors extrapolate the speculative, yet physically viable, conjecture that particle radius varies with the cube root of the energy available at each discharge step.

Guided by these considerations we assume that the coefficient $\gamma$, in the recursive energy modeling equation, can be expressed as a function of $\sqrt[3]{E_{k}}$ :

$E_{k+1}=f\left(E_{k}\right)$, with $\quad f(E)=r \sqrt[3]{E}(1-E)$

in which $r$ takes into account the modes of debris distribution and bridge formation, as well as the effect of gas bubbles. Bridges can be either formed by chains or by clusters, as shown in Figure 6. Chains correspond to the lowest number of particles necessary to obtain a bridge (small gap, high electric field, alignment of the particles along the force lines, low energy required for bridge formation). In this case, the time required for bridge formation is probably short and an early discharge ignites before the voltage can increase to the nominal value. On the other hand, clusters are likely to require longer ignition delays and to associate with higher discharge efficiency (i.e. a discharge energy that is close to the theoretical one). Packing of particles into clusters requires time during which the discharge voltage approaches the nominal value.

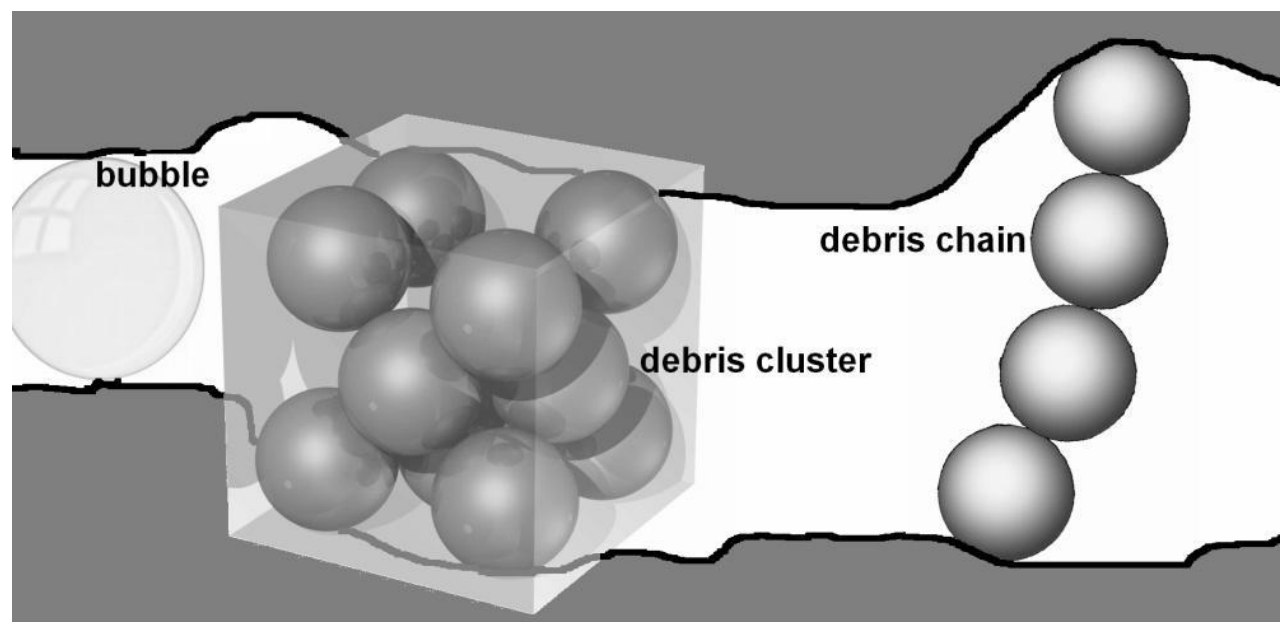

Figure 6: Modes of formation of debris bridges. (Left) Maximum density: bridge due to the maximum number of spheres that can be packed together in a small volume, i.e. a cluster. (Right) Bridge produced by the lowest number of particles that are aligned along the force lines of the electric field. 


\section{Basics on deterministic chaos}

Basic concepts of chaos theory, instruments and terminology are briefly reviewed here, as a support to the analysis of model behavior in the following section.

One of the hallmarks of chaos is a deterministic evolution with unpredictable outcome. Despite the fact that a system evolves according to a simple deterministic rule, forecasting the future of the system can be essentially impossible [11] The logistic map is a classical model in chaos theory. It is the discrete version of the logistic equation, which is a model of population growth. It is the parametric quadratic recurrence equation (6), capable of a very complicated behavior.

$x_{k+1}=f\left(x_{k}\right)$, with $f(x)=r(x)(1-x)$

Depending on the parameter $r$, the equation shows different qualitative behaviors: regular, or oscillatory, or chaotic. A visual tool called Web, Cobweb, or Verhulst diagram, is used to investigate the qualitative behaviour of onedimensional iterated functions, such as the logistic map. Using a Verhulst diagram, it is possible to infer the long term status of an initial condition $\mathrm{x}_{0}$ under repeated application of a map. For a given iterated function $f: \mathrm{R} \rightarrow \mathrm{R}$, the web diagram consists of a diagonal line $(y=x)$ and a curve representing $y=f(x)$ : the intersections are the fixed points or attractors of $f(x)$, that is to say points where the sequence of iterates converges. An attractor contains information about the long term dynamical behaviour of the system. On the Verhulst plot:

- a stable fixed point corresponds to an inward spiral;

- an unstable fixed point is an outward one.

It follows from the definition of a fixed point that these spirals will center at a point where the diagonal line $(y=x)$ crosses the function graph.

- A period 2 orbit is represented by a rectangle, while greater period cycles produce further, more complex closed loops. - A chaotic orbit would show a 'filled out' area, indicating an infinite number of non-repeating values located on a socalled strange attractor (also called chaotic, as it is an attractor for which the approach to the final set of physical properties is chaotic).

The shift between different qualitative behaviors, occurring at certain values of the parameter $r$, is called bifurcation. The logistic map shows subsequent bifurcations, each doubling the number $2^{\mathrm{n}}$ of fixed points, called period doubling bifurcations. As $n$ tends to infinity, the values of $r$ where the period doubling bifurcations occur tend to a value called accumulation point, at which periodicity gives way to chaos. In the middle of this complexity, a window suddenly appears with a period 3, then period doubling begins again, eventually leading to a new chaotic state. Li and Yorke proved that any one-dimensional system which exhibits a regular 3-cycle will also display regular $n$-cycles of every order $n$, as well as completely chaotic orbits [33]. 
Development of the recursive series from two different nearby initial conditions leads to two orbits. The divergence between the two trajectories can be expressed as $e^{\lambda x}$, in which $\lambda$ is the Lyapunov exponent. In other terms, $\lambda$ characterizes the asymptotic rate of separation of nearby trajectories. A dynamic system is chaotic if at least one of the Lyapunov exponents, calculated in each iteration during the system evolution, is positive. The latter implies that the system is sensitive to small perturbations in the initial conditions that will be amplified yielding totally different orbits; as a consequence forecasting of the system evolution rapidly becomes impossible.

An interesting feature of the sequence of period $2^{\mathrm{n}}$ bifurcation points is that ratios of differences of successive iterates converge to the Feigenbaum constant $\delta=4.6692$. For functions approaching chaos via period doubling, $\delta$ characterizes the geometric approach of the bifurcation parameter to its limiting value.

\section{Analysis of model behavior}

The aim of this section is to demonstrate that Equation (5) has similar chaotic behaviour to the ubiquitous Logistic map. Our investigation makes use of numerical and algebraic capabilities of Mathematica, described by Sofroniou and colleagues [27-29] and more generally in Mathematica [30].

Three time series for the proposed model for EDM are shown in Figure 7. Using the same initial energy but different parameter values, the behaviour is completely different. Figure 8 gives an alternative illustration of Equation (5), with the Verhulst diagram. With $r=1.5$ the iterations converge to a stable attractor, whereas with $r=1.8$, the iterations settle into a stable 2-cycle. This suggests that the system has undergone a bifurcation. With $r=2.1$, the trajectory appears to be chaotic. In this case, given any value of $\mathrm{E}_{\mathrm{k}}, \mathrm{E}_{\mathrm{k}+1}$ can not be determined, as it can be anywhere on the red curve that becomes a strange attractor.
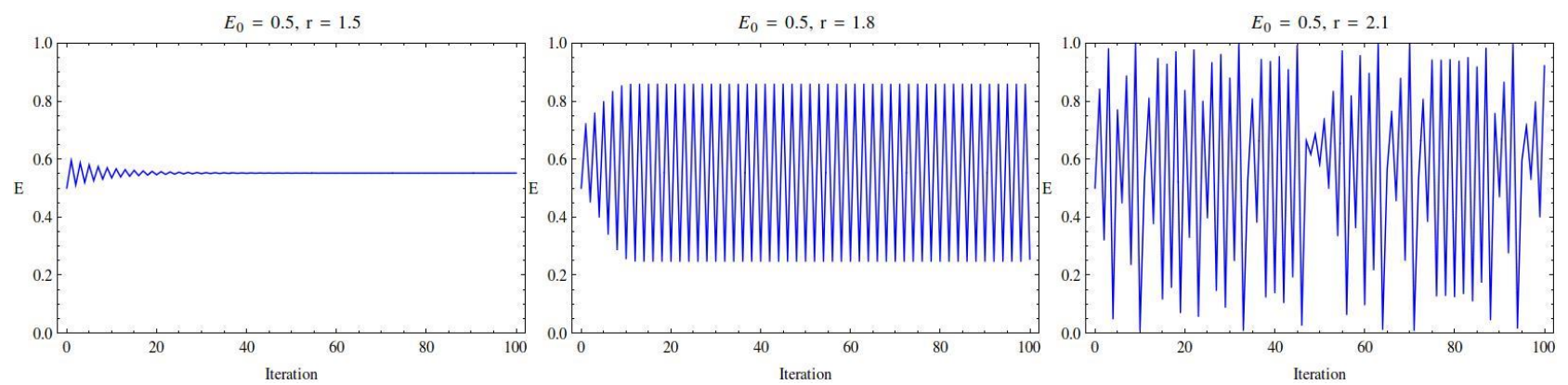

Figure 7. Evolution of three time series for the EDM model proposed in Equation (5). 

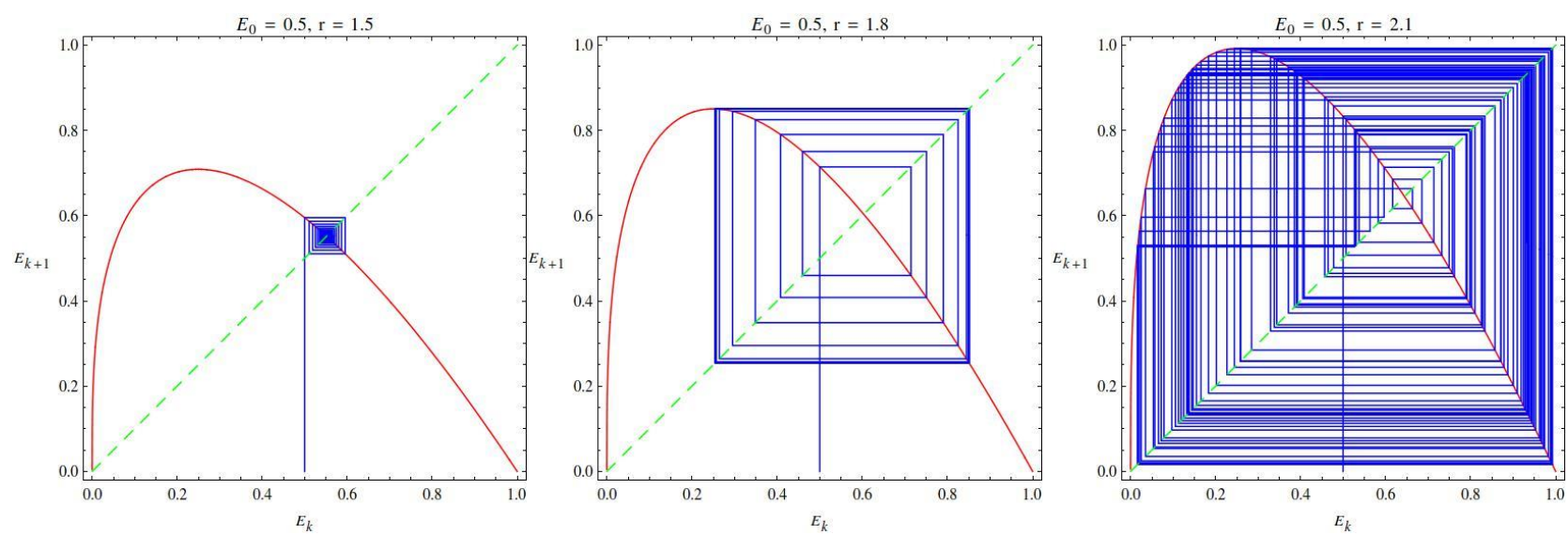

Figure 8. Verhulst diagrams for Equation (5). Convergence to a fixed point on the left, a 2-cycle in the middle and a chaotic trajectory on the right.

Ignition of a first discharge with the same initial energy $E_{0}$ but different values of the parameter $r$, then, seems to lead to different energy possibilities or, in other terms, different discharge types. For an initial value $E_{0}=0.5$, for example, Figures 7 and 8 show that subsequent energy values $E_{k}$ may all tend to the initial value (when $\mathrm{r}=1.5$ ), or oscillate between a minimum and a maximum value (when $r=1.8$ ), or take an unpredictable value in a chaotic series (when $r=$ 2.1).

To explain the previous situation, consider the iteration of a function $f$ at an initial value $E_{0}=E$ as $E_{i+1}=f\left(E_{i}\right), i>0$. The Lyapunov exponent $\lambda$ characterizes the asymptotic rate of separation of nearby trajectories. For practical estimation the first $m$ iterations are not taken into consideration and a finite approximation is computed:

$\lambda=\lim _{n \rightarrow \infty} \sum_{i=1}^{n} \frac{\log \left(\mid f^{\prime}\left(E_{i} \mid\right)\right.}{n} \approx \sum_{i=m+1}^{n} \frac{\log \left(\mid f^{\prime}\left(E_{i} \mid\right)\right.}{(n-m)}$

For the remaining figures in this section we have chosen $m=10^{4}$ and $n=2 m$, which was found to be accurate enough for graphical purposes. For $\lambda<0$ small changes in the starting values diminish, while for $\lambda>0$ they are amplified. When $\lambda=0$, bifurcations in the behaviour of orbits take place. The bifurcation diagram and Lyapunov exponent for Equation (5) are shown in Figure 9. 

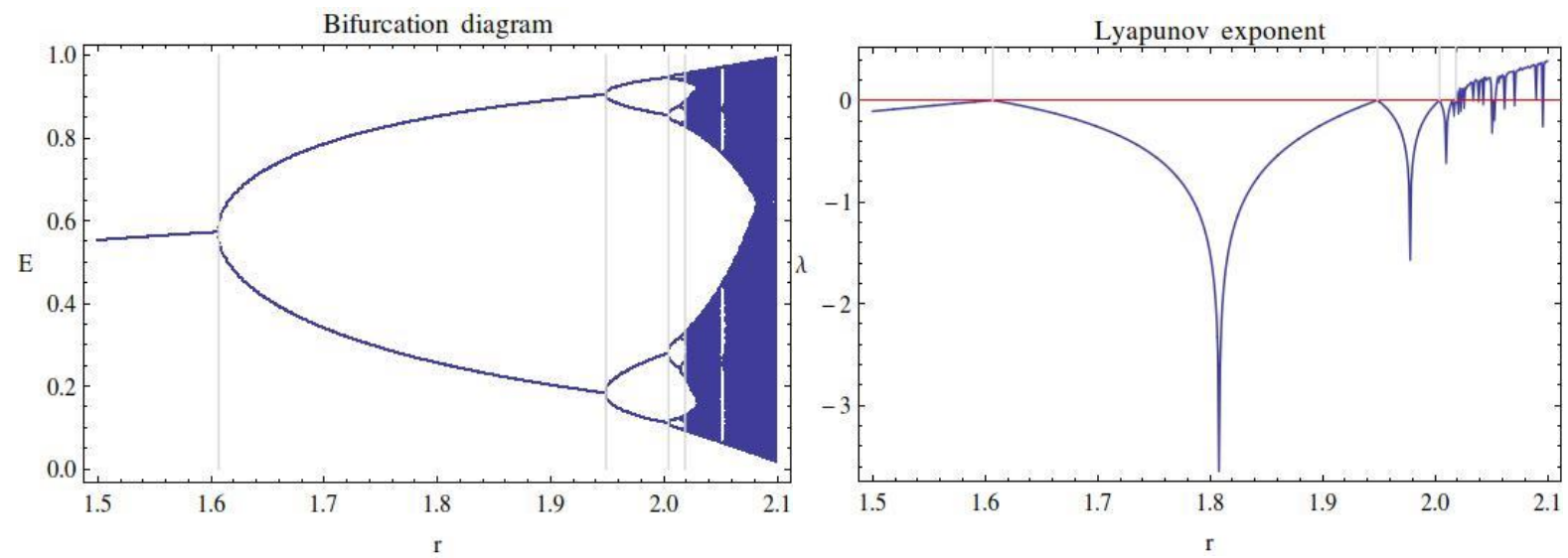

Figure 9. Bifurcation diagram and numerical approximants for the Lyapunov exponent for Equation (5).

Let $r_{n}$ denote a value of the parameter $r$ which determines the onset of a period $n$-cycle, or bifurcation point of order $n$. The location of $r_{n}$ can be obtained by solving the following system of $n+1$ equations [31]:

$$
\begin{array}{ccc}
E_{1} & = & f\left(E_{0}\right) \\
& \vdots & \\
E_{n-1} & = & f\left(E_{n-2}\right) \\
E_{0}=E_{n} & = & f\left(E_{n-1}\right) \\
\left|\prod_{i=1}^{n} f^{\prime}\left(E_{i-1}\right)\right| & = & 1
\end{array}
$$

with $f$ given by Equation (5). The first $n$ equations give $E_{l}, E_{2}, \ldots, E_{n}$ (where $E_{n}=E_{0}$ ). The last equation uses the fact that the onset of period $n$ occurs by a fold bifurcation, so that the $n^{\text {th }}$ derivative is 1 . This expresses the fact that the bifurcation point is the right endpoint of the interval for the parameter $r$ for which the period- $n$ solution is stable. We have used Gröbner Bases [32] in Mathematica to find exact solutions to Equations (8) for small $n$ (the complexity rapidly increases with $n$ ). Essentially, a lexicographical Gröbner basis can be used to obtain a univariate polynomial in the parameter $r$. This univariate polynomial can then be used to find all the solutions of the system.

Solving Equations (8) we obtained a polynomial of degree 123 in $r$, for which we found a real solution calculated by numerical approximation $r_{3} \approx 2.11487$. The latter proves the onset of a period 3 orbit. Due to the results from Li and Yorke [33], this solution provides a (computer assisted) proof that our EDM model can indeed exhibit chaos.

The first few period $2^{n}$ bifurcation points for Equation (5), $r_{2} n$, are given numerically in Table 1. The Lyapunov exponent becomes positive at the accumulation point, $r_{\infty}$, where periodic orbits give way to chaotic ones. The study of our proposed recursive energy model (5), carried out so far, shows that, for a same choice $0<E_{0}<1$ of the initial 
energy, values of the parameter $r$ that are greater than $r_{\infty}$ lead to successive energy values that possess chaotic features. Forecasting of the discharge energy even with constant input parameters can thus be impossible when the presence of debris is relevant.

Table 1. Numerical approximations to bifurcation points for Equation (5).

\begin{tabular}{cccc}
\hline $\mathrm{n}$ & $2^{\mathrm{n}}$-cycle & $r_{2^{n}}$ & $\delta_{n}$ \\
\hline 1 & 2 & 1.6067615 & \\
2 & 4 & 1.9488519 & \\
3 & 8 & 2.0040855 & 6.19352 \\
4 & 16 & 2.0155541 & 4.81607 \\
5 & 32 & 2.0179981 & 4.69255 \\
6 & 64 & 2.0185210 & 4.67393 \\
7 & 128 & 2.0186329 & 4.67292 \\
\hline \multirow{4}{*}{ accumulation } & 2.0186648 & \\
& point & & \\
\hline
\end{tabular}

The bifurcation points $r_{2}, r_{4}, r_{8}$ and $r_{\infty}$ are highlighted in Figure 9 as vertical gray lines. The last column of Table 1 shows that the ratios of differences of successive iterates $\delta_{n}$ converge to the Feigenbaum constant $\delta$ :

$\delta=\lim _{n \rightarrow \infty} \frac{\left(r_{2^{n+1}}-r_{2} n\right)}{\left(r_{2^{n+2}}-r_{2^{n+1}}\right)}=\lim _{n \rightarrow \infty} \delta_{n} \approx 4.6692$

The Feigenbaum constant is universal if the Schwarzian derivative $(S f)(x)$ is negative in a bounded interval [34], so that for Equation (5):

$(S f)(x)=\frac{f^{\prime \prime \prime}(x)}{f^{\prime}(x)}-\frac{3}{2}\left(\frac{f^{\prime \prime}(x)}{f^{\prime}(x)}\right)^{2}=-\frac{4\left(14 x^{2}+14 x-1\right)}{9 x^{2}(4 x-1)^{2}} \quad$ if $r \neq 0$,

which holds for

$x \in\left[\frac{3 \sqrt{7}-7}{14}, 1\right] \approx[0.06694671,1]$

Figure 10 illustrates the asymptotic dynamical behaviour of Equation (5) in the realm of chaos, showing a strange attractor in two and three-dimensional space. Unlike other types of attractors, on a chaotic attractor it is not possible to determine exactly where the system will be. Furthermore, chaotic attractors never close on themselves. In other words, motion on chaotic attractors never repeats and it is chaotic. 

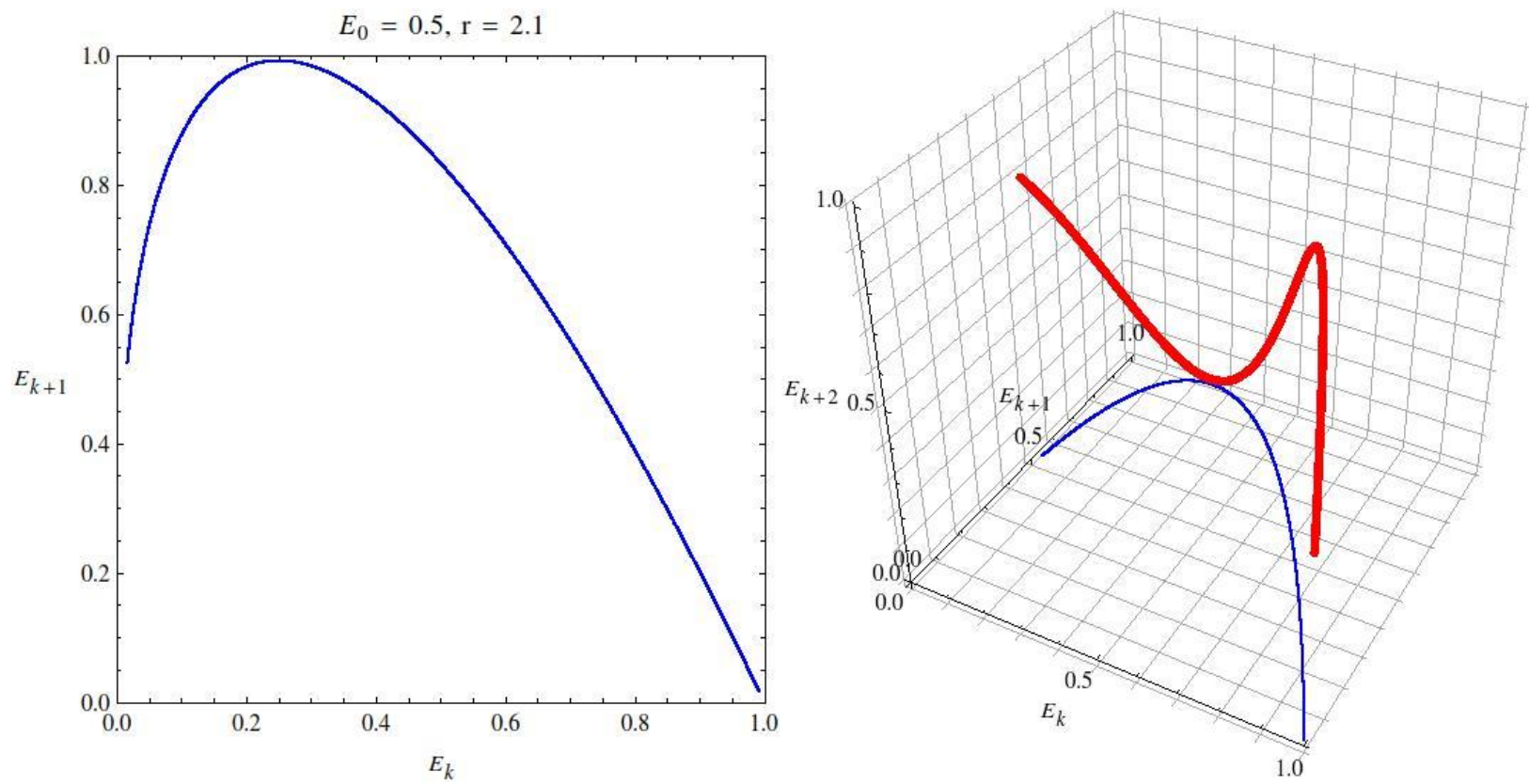

Figure 10. Asymptotic dynamical behaviour of Equation (5). Chaotic attractor in two dimensions (left) and three dimensions (right). The 2D plane projection in 3D corresponds to the leftmost picture.

\section{Conclusions}

The paper builds on a new model for EDM processes centered on discharge ignition via debris bridges. Early experimental clues questioning the thermoelectric theory and recent direct proofs of bridge formation are merged into a comprehensive view, where each process step is deterministically related to the previous one, that is however unknown, so that the overall evolution is unpredictable.

The role of debris in discharge ignition couples each EDM step to the previous spark in a recursive series, where the effects of the process history determine the efficiency of the current discharge. A recursive model is proposed here, based on experimental observations, for the machining energy employed at each discharge event. Behavior of the model is studied and shows a chaotic evolution similar to that of the Logistic map. A chaotic attractor for our model can also be determined.

Our analysis demonstrates that, starting from any choice of the (EDM process) parameters, the process can evolve towards a situation described by a (chaotic) attractor. It can be concluded that, in EDM, chaos not only is observed in the location of discharges, but it characterizes the discharge energy as well. Such an interpretation explains why it is possible that various types of discharge can take place, with differing machining efficiency, under the same conditions imposed by the process parameters.

Current control units work by taking the system back to the pre-chaotic state, but new control approaches might perhaps be developed in the wake of the findings of this research. 


\section{Appendix}

One of the most widely used EDM models is illustrated in Figure A.11: discharges occur by ion action at the overcoming of the dielectric breakdown strength. A plasma channel is generated where the electrons move faster than the ions [3]. The anode starts melting first, resulting in a larger molten area than at the cathode. As time advances, the anode will begin to resolidify (after a few microseconds), due to the decrease in the local heat flux at the anode surface, while the molten area in the cathode is still expanding. Hence, the molten volume at the anode will be smaller than at the cathode.

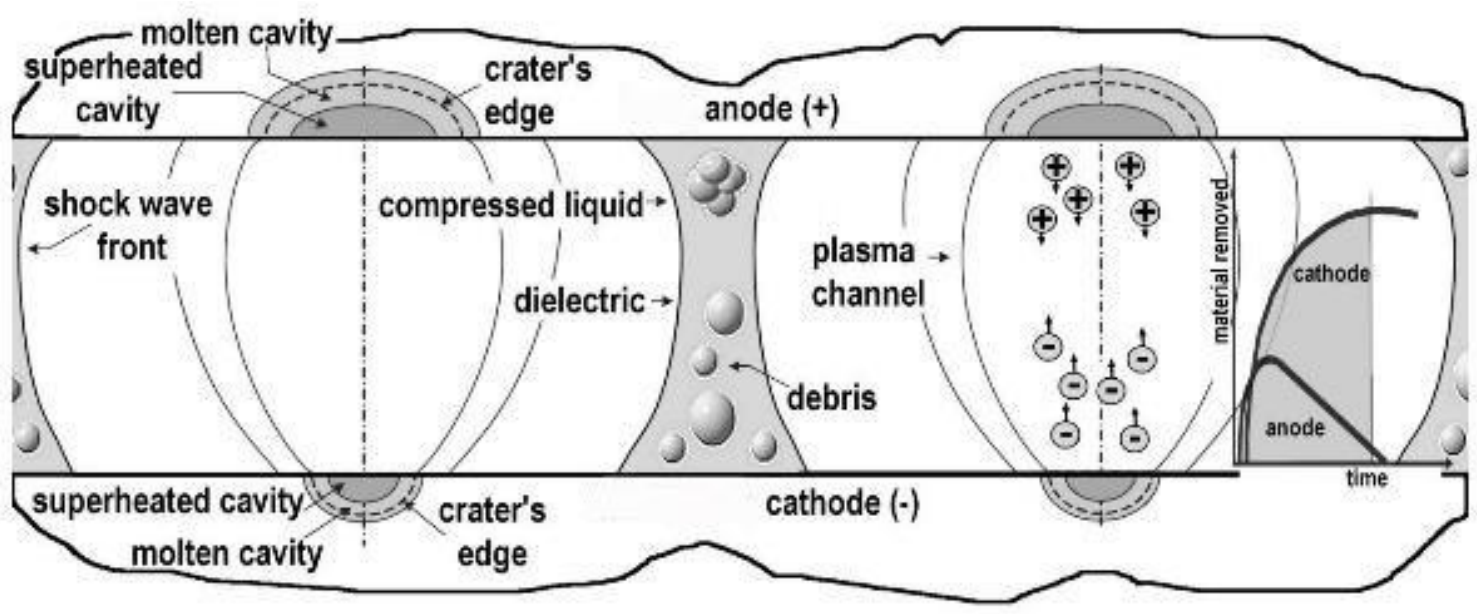

Figure A.11. Common model used to explain the EDM mechanism.

However, this kind of model does not give explanation to any of the following issues.

- $\quad$ Even with a stable process set-up, different kinds of sparks in a wide range of energies are obtained, which cause marked variations in height and shape of the recast layer on an electro-machined surface (see Figure A.12 as an example).

- $\quad$ Location of sparks has been found to be chaotic rather than random by Han and Kunieda [10].

- $\quad$ Complex phenomena occur in the gap, moving away from the ideal uncontaminated state (for which the thermoelectric model can be exhaustive). These phenomena firstly suggested the need for a new model. We collect them in the following list $(\mathrm{a} \div \mathrm{f})$ providing a brief description for each item. 


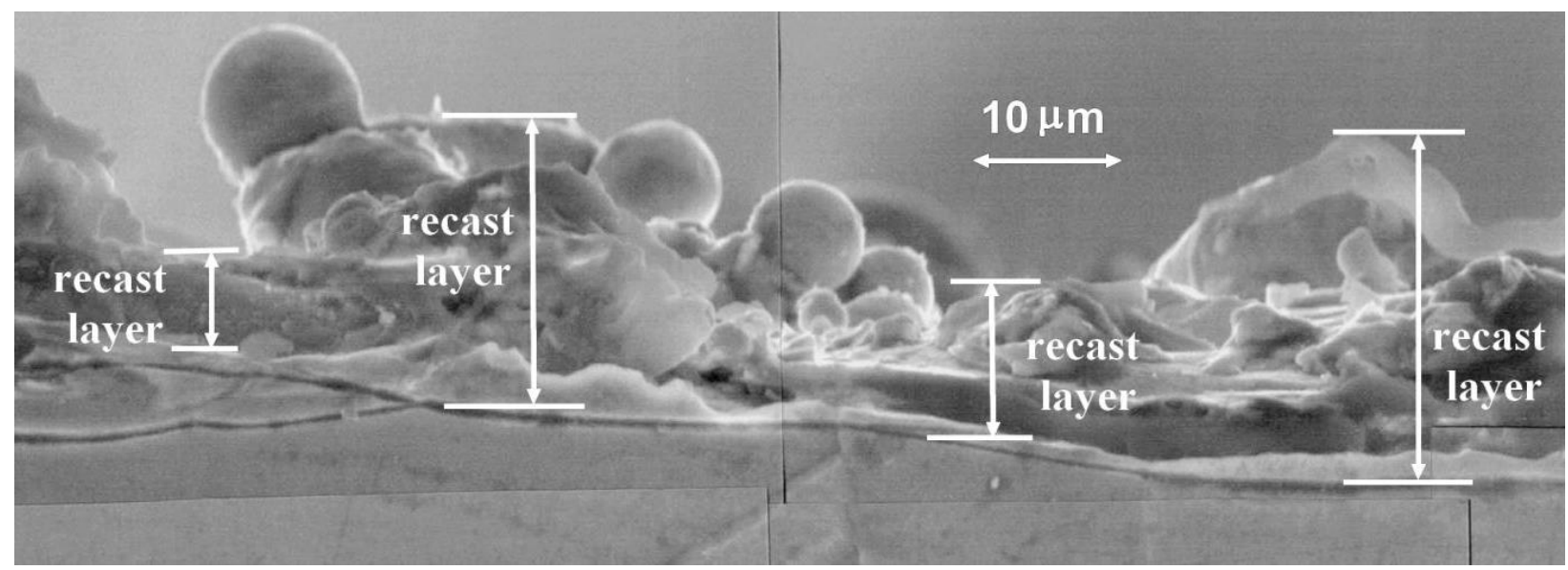

Figure A.12. Cross section of a wire electrical discharge machined surface of Inconel 718, machined using $120 \mathrm{~V}$ voltage, 24A current intensity, $4 \mu$ s pulse-on duration, $50 \mathrm{kHz}$ frequency. Height and shape variation in the recast layer can be observed.

\section{a. Insulating effect of the dielectric changes due to temperature}

Light et al. inspected the effect of temperature on the conductivity of water [35]. They found that at $0^{\circ} \mathrm{C}$ the sensitivity of conductivity (or resistivity) of ultra-pure water to temperature is $7.4 \%$ per ${ }^{\circ} \mathrm{C}$. At $100^{\circ} \mathrm{C}$ this sensitivity drops (by a factor of three) to $2.3 \%$ per ${ }^{\circ} \mathrm{C}$. Within the gap in an EDM process, the temperature changes continuously and unpredictably, thus the dielectric breakdown strength in not uniquely determined.

\section{b. Dielectric strength increases when flowing dielectric liquid is used}

The dielectric breakdown statistics change when the stressed liquid moves relative to the electrodes and that the basic phenomena are independent of the degree of particle contamination [36]; equivalently, all liquids contain an unperceivable background contamination, causing them to behave in a similar fashion.

\section{c. Gas bubbles hinder the dielectric flow in the gap}

The movement of the debris is influenced by gas bubbles, arising from evaporation and sometimes by chemical reaction of the dielectric fluid at ignition, as well as by geometrical gap conditions [6].

\section{d. Dielectric strength changes in time}

The dielectric strength substantially changes in time, as a consequence of the erosion process, which contaminates the dielectric itself. When a fresh dielectric liquid is exposed to sparking, during machining, its dielectric strength is reduced by many orders of magnitude, due to the eroded particles that become suspended in the liquid. This effect is crucial for EDM, since if it did not occur then much higher energies per discharge would be needed. During the EDM process the dielectric is filtered, but filtering efficiency differs from the ideal unit value; therefore the presence of small particles, produced during the previous discharges, is to be expected. An estimate of the distribution of the particles has 
been attempted in [8]. Furthermore, due to the variability of the local gap thickness, sinks can be present in which the particles accumulate.

\section{e. $\quad$ Ignition delay time depends on the gap condition}

Since dielectric breakdown strength decreases with the decrease of the gap width and increase of the density of debris particles, the ignition delay time depends on the gap conditions. The effect is well described by Kunieda et al. [11].

\section{f. $\quad$ Debris particles produce bridges between electrodes}

In EDM, since the dielectric liquid is contaminated with electrically conductive debris particles, their effect in the gap should be taken into account. An activation or delay time is required in order for debris particles to form bridges between the electrodes [11]. Several papers investigate the ignition mechanism of the discharge and they highlight the debris bridge as a driving phenomenon for the discharge process, as first postulated by Schumacher [5]. As a consequence, the pulse durations are directly proportional to the extent of contamination in the gap.

If the proposed mechanism of ignition via particle bridges is accepted, the question immediately arises as to how quickly such particles can be arranged in a line. This depends on dimension, number and size distribution of the particles: the bubbles might cause the formation of locally occluded cells, that prevent the efficient removal of debris. Ignition can thus occur either by ionization across the residual distances shortened by debris, for which much higher field strengths exist, or by evaporation of the bridge under excessive current. A recent paper provided proof of the role of debris in the ignition of discharges and distinguished two modes of bridge formation by chains or by clusters [8].

\section{References}

[1] Ho KH, Newman ST (2003) State of the art electrical discharge machining (EDM). Int J Mach Tools Manuf 43(13): 1287-1300.

[2] Yeo SH, Kurnia W, Tan PC (2008) Critical assessment and numerical comparison of electro-thermal models in EDM. J Mat Process Tech 203(1-3): 241-251

[3] Yeo SH, Kurnia W, Tan PC (2007) Electro-thermal modelling of anode and cathode in micro-EDM. J Phys D: Appl Phys 40(8):2513-2521

[4] Schumacher BM (2004) After 60 years of EDM the discharge process remains still disputed. J Mat Process Tech 149(81-3): 376-381.

[5] Schumacher BM (1990) About the Role of Debris in the Gap During Electrical Discharge Machining. CIRP Ann Manuf Technol 39(1):197-199.

[6] Schulze HP, Mecke K, Wollenberg G (2005) Influence of gas bubbles on electrical discharges in small working gaps. International Conference on Dielectric Liquids. ICDL 2005, 63- 66 
[7] Luo YF (1997) The dependence of interspace discharge transitivity upon the gap debris in precision electrodischarge machining. J Mat Process Tech 68(2):121-131.

[8] Gatto A, Bassoli E, Denti L, Iuliano L (2013) Bridges of debris in the EDD process: going beyond the thermoelectrical model. J Mat Process Tech 213(3):349-360.

[9] Pandit SM, Rajurkar KP (1983) A stochastic approach to thermal modelling applied to electro-discharge machining. Trans ASME J Heat Trans 105:555-558.

[10] Han F, Kunieda M (2001) Chaos Found in Distribution of EDM Spark. Proc of ISEM XIII, 185-192.

[11] Kunieda M, Lauwers B, Rajurkar KP, Schumacher BM (2005) Advancing EDM through Fundamental Insight into the Process. CIRP Ann - Manuf Technol 54(2): 64-87.

[12] Salonitis K, Tournaras A, Stavropoulos P, Chryssolouris G (2009) Thermal modeling of the material removal rate and surface roughness for die-sinking EDM. Int J Adv Manuf Technol 40:316-323.

[13] Adineh VR (2012) Radiative Heat Loss of Electrical Discharge Machining Process Through Hydrocarbon Oil and Deionized Water Dielectric Liquids. Plasma Chem Plasma P 32(2):369-392.

[14] Jilani ST, Pandey PC (1982) Analysis and modelling of EDM parameters. Precis Eng 4(4):215-221.

[15] Van Dijck F (1973) Physico-Mathematical Analysis of the Electro Discharge Machining Process. Dissertation of Katholieke Universiteit Leuven.

[16] Patel MR, Barrufet MA, Eubank PT, DiBitonto DD (1989) Theoretical Models of the Electrical Discharge Machining Process II. The Anode Erosion Model. J Appl Phys 66(9):4104-4111.

[17] Xia H, Hashimoto H, Kunieda M, Nishiwaki N (1996) Measurement of Energy Distribution in Continuous EDM Process, Seimitsu Kogaku Kaishi/J Jpn Soc Prec Eng 62(8):1141-1145.

[18] Behrens AW, Ginzel J, Bruhns F-L (2004) Threshold technology and its application for gap status detection. J Mat Process Tech 149(1-3):310-315.

[19] Ginzel J, Behrens AW, Wulfsberg J-P (2004) Technology development for EDM using statistical analysis of arcing information. J Mat Process Tech 149(1-3):232-237.

[20] Dauw D, Snoeys R (1986) On the Derivation and Application of a Real-Time Tool Wear Sensor in EDM. CIRP Ann - Manuf Technol 35(1):111-116.

[21] Dauw DF, Snoeys R, Dekeyser W (1983) Advanced Pulse Discriminating System for EDM Process Analysis and Control. CIRP Ann - Manuf Technol 32(2):541-549.

[22] Jennes M, Snoeys R, Dekeyser W (1984) Comparison of Various Approaches to Model the Thermal Load on the EDM-Wire Electrode. CIRP Ann - Manuf Technol 33(1):93-98. 
[23] Frei C, Hirt C, Girardin R, Dauw DF (1987) A New Approach for Contamination Measurements for EDM Dielectrics, CIRP Ann - Manuf Technol 36(1):111-113.

[24] Calignano F, Denti L, Bassoli E, Gatto A, Iuliano L (2012) Studies on electro discharge drilling of an Al2O3 -TiC composite. Int Journal Adv Manuf Technol 66(9):1757-1768.

[25] Tebni W, Boujelbene M, Bayraktar E, Ben Salem S (2009) Parametric approach model for determining electrical discharge machining (edm) conditions: effect of cutting parameters on the surface integrity. Adv Mat Res 83-86:725737.

[26] Zhang HT, Lee C, Lau WS (1997) Study on the electro-discharge machining of a hot pressed aluminum oxide based ceramic. J Mat Process Tech 63:908-912.

[27] Sofroniou M (1996) Numerics in Mathematica 3, Mathematica Journal 6(4):64-73.

[28] Knapp R, Sofroniou M (1997) Difference Equations and Chaos in Mathematica, Dr. Dobbs Journal 22(11):84-90.

[29] Sofroniou M, Spaletta G (2005) Precise numerical computation. J Log Algebraic Program 64(1):113-134.

[30] Mathematica, Version 9.0, Wolfram Research Inc., Champaign, Illinois, USA, 2011. www.wolfram.com

[31] Strogatz SH (1994) Nonlinear Dynamics and Chaos, Addison-Wesley, Reading, MA, USA.

[32] Buchberger B (1976) Theoretical Basis for the Reduction of Polynomials to Canonical Forms, SIGSAM Bulletin 39:19-24.

[33] Li TY, Yorke JA (1975) Period Three Implies Chaos. American Mathematical Monthly 82:985-992.

[34] Tabor M (1989) Chaos and Integrability in Nonlinear Dynamics: An Introduction, Wiley, New York, 220.

[35] Light TS, Licht S, Bevilacqua AC, Morashc KR (2005) The Fundamental Conductivity and Resistivity of Water, Electrochemical and Solid-State Letters 8(1):E16-E19.

[36] Bommeli C, Frei C, Ratajski A (1979) On the influence of mechanical perturbation on the breakdown of a liquid dielectric. Journal of Electrostatics 7:123-144. 


\section{Figure captions}

Figure 1. Energy distribution in the discharge area.

Figure 2. Categorization of pulse types: short circuits, arcs, normal discharges and open circuits.

Figure 3. FIB-sectioned debris produced in small-hole $\mathrm{ED}$ drilling on a $72 \mathrm{wt} \% \mathrm{Al}_{2} \mathrm{O}_{3}-28 \mathrm{wt} \% \mathrm{TiC}$ composite.

Figure 4. Examples of debris chains and clusters produced in small-hole ED drilling on a $72 \mathrm{wt}_{\%} \mathrm{Al}_{2} \mathrm{O}_{3}-28 \mathrm{wt} \% \mathrm{TiC}$ composite.

Figure 5. Scheme of EDM pulse times.

Figure 6: Modes of formation of debris bridges. (Left) Maximum density: bridge due to the maximum number of spheres that can be packed together in a small volume, that is a cluster. (Right) Bridge produced by the lowest number of particles that are aligned along the force lines of the electric field.

Figure 7. Evolution of three time series for the EDM model proposed in Equation (5).

Figure 8. Verhulst diagrams for Equation (5). Convergence to a fixed point on the left, a 2-cycle in the middle and a chaotic trajectory on the right.

Figure 9. Bifurcation diagram and numerical approximants for the Lyapunov exponent for Equation (5).

Figure 10. Asymptotic dynamical behaviour of Equation (5). Chaotic attractor in two dimensions (left) and three dimensions (right). The 2D plane projection in 3D corresponds to the leftmost picture.

Figure A.11. Common model used to explain the EDM mechanism.

Figure A.12. Cross section of a wire electrical discharge machined surface of Inconel 718, machined using $120 \mathrm{~V}$ voltage, 24A current intensity, $4 \mu$ s pulse-on duration, $50 \mathrm{kHz}$ frequency. Height and shape variation in the recast layer can be observed.

\section{Table captions}

Table 1. Numerical approximations to bifurcation points for Equation (5). 